\title{
Translocation Breakpoints in FHIT and FRA3B in Both Homologs of Chromosome 3 in an Esophageal Adenocarcinoma
}

\author{
Jianming M. Fang, ${ }^{1,2}$ Martin F. Arlt, ${ }^{1,2}$ Ann C. Burgess,' Susan L. Dagenais, ${ }^{1,2}$ David G. Beer, ${ }^{3}$ \\ and Thomas W. Glover ${ }^{1,2 *}$ \\ 'Department of Pediatrics, University of Michigan, Ann Arbor, Michigan \\ ${ }^{2}$ Department of Human Genetics, University of Michigan, Ann Arbor, Michigan \\ ${ }^{3}$ Department of Surgery, University of Michigan, Ann Arbor, Michigan
}

Common fragile sites have been proposed to play a mechanistic role in chromosome translocations and other rearrangements in cancer cells in vivo based on their behavior in vitro and their co-localization with cancer translocation breakpoints. This hypothesis has been the subject of controversy, because associations have been made at the chromosomal level and because of the large number of both fragile sites and cancer chromosome breakpoints. Tests of this hypothesis at the molecular level are now possible with the cloning of common fragile site loci and the use of fragile site clones in the analysis of rearranged chromosomes. FRA3B, the most frequently seen common fragile site, lies within the large FHIT gene. It is now well established that this region is the site of frequent, large intragenic deletions and aberrant transcripts in a number of tumors and tumor cell lines. In contrast, only one tumor-associated translocation involving the FHIT gene has been reported. We have found translocations in both homologs of chromosome 3 in an early-passage esophageal adenocarcinoma cell line. This cell line showed no normal FHIT transcripts by reverse transcription polymerase chain reaction. Subsequent chromosome analysis showed translocations of the short arms of both homologs of chromosome $3: t(3 ; 16)$ and $t(3 ; 4)$. The breakpoints of both translocations were shown by fluorescence in situ hybridization and polymerase chain reaction to be in the FHIT gene, at or near the center of the fragile site region. Using rapid amplification of cDNA ends with FHIT primers, a noncoding chimeric transcript resulting from $\mathrm{t}(3 ; 16)$ was identified. These data provide direct support for the hypothesis that FRA3B, and likely other common fragile sites, may be "hot spots" for translocations in certain cancers, as they are for deletions, and that such translocations have the potential to form abnormal chimeric transcripts. In addition, the results suggest selection for loss of a functional FHIT gene by the translocation events.

() 200I Wiley-Liss, Inc.

\section{INTRODUCTION}

It is well established that common fragile sites are loci that are especially sensitive to forming gaps or occasionally breaks on metaphase chromosomes when cultured with aphidicolin or under conditions of folate stress (Glover, 1998). Consistent with a possible role in chromosome rearrangements in vivo, they also have been shown in cells cultured in vitro to be "hot spots" for translocations and deletions (Glover and Stein, 1988; Wang et al., 1997), sister-chromatid exchanges (SCEs) (Glover and Stein, 1987), DNA integration (Rassool et al., 1991), and DNA breaks associated with gene amplification in CHO cells (Coquelle et al., 1997). The association between the location of common fragile sites and sites of chromosome translocation or other rearrangements in cancer and leukemia was noted by many investigators when common fragile sites were first characterized (Glover et al., 1984; Hecht and Glover, 1984; Le Beau and Rowley, 1984; Yunis and Soreng, 1984). This coincident location of fragile sites and recurrent cancerrelated breakpoints led to the hypothesis that there is a mechanistic relationship between the two. This hypothesis was advanced most noticeably by Yunis and Soreng (1984), who promoted it by noting a large number of such associations in numerous leukemias, lymphomas, and solid tumors. This hypothesis, however, has been the subject of controversy, owing to the sheer number of both breakpoints and fragile sites and the fact that the location of fragile sites was based on conventional cytogenetic assignments and not on molecular maps.

The cloning of common fragile site regions has allowed more sensitive molecular analysis of their behavior in cancer cells. The FRA3B fragile site lies within the FHIT gene, which has been shown to be mutated by large intragenic deletions and to produce aberrant transcripts in a variety of tumor

\footnotetext{
Supported by: National Cancer Institute; Grant number: CA43222.

*Correspondence to: Dr. Thomas W. Glover, Department of Human Genetics, Box 0618, 4909 Buhl, University of Michigan, Ann Arbor, MI 48109-0618. E-mail:glover@umich.edu

Received 7 September 2000; Accepted 11 September 2000
} 
cells (Sozzi et al., 1998). The deletions suggest that instability at the fragile site is mechanistically responsible for the deletions and also that there is selection for loss of functional FHIT alleles in these tumor cells. Recent investigations of other common fragile sites show a similar pattern of deletion in cancer cells, in agreement with the hypothesis that this is a general feature of common fragile sites (Huang et al., 1999; Mangelsdorf et al., 2000; Paige et al., 2000). For the most part, the rearrangements studied and reported to date are large deletions of hundreds of kilobases.

Few attempts have been made to identify translocations or other gross chromosomal rearrangements at fragile sites, which, if found, would support original suggestions concerning fragile sites and cancer-related chromosome rearrangements. Only two translocations have been reported previously, with breakpoints shown to be in the FHIT/ FRA3B region. One of these, $\mathrm{t}(3 ; 8)(\mathrm{p} 14.2 ; \mathrm{q} 24)$, was a constitutional translocation associated with hereditary renal cell cancer in one family (Cohen et al., 1979), which was found to result in a chimeric transcript between FHIT and the TRC 8 gene, a putative patched family member (Gemmill et al., 1998). The second was in a pleomorphic adenoma of the thyroid gland, which resulted in the fusion of FHIT and the HMGIC gene on chromosome 12 (Geurts et al., 1997). Recently, the FRA16D region has been shown to contain a gene termed FOR, with homology to the oxidoreductase gene family, within which three of five previously mapped multiple myeloma translocation breakpoints are located (Mangelsdorf et al., 2000).

We report here the finding of two different translocation breakpoints within FRA3B and the FHIT gene in both homologs of chromosome 3 in an early-passage esophageal adenocarcinoma cell line. Characterization of the two translocations showed one to result in a nonfunctional chimeric transcript with sequences at $16 \mathrm{p} 13.3$. The second is a translocation to a region on distal $4 \mathrm{p}$. These results provide further evidence that fragile sites participate in translocations in tumor cells. Together with earlier findings of translocations within fragile site regions in cancers, they suggest that such events may not be infrequent, and they provide a mechanism for gene loss or formation of novel transcripts.

\section{MATERIALS AND METHODS}

\section{Cell Lines}

The BIC-1 adenocarcinoma cell line was established from minced tumor tissue obtained from a male patient who had undergone surgical resection for a stage T3, N1, MO Barrett adenocarcinoma and who had received no other preoperative treatments, such as radiation or chemotherapy. The cell line was grown in Dulbecco modified Eagle medium with $15 \%$ fetal bovine serum and analyzed at passages $8-10$.

\section{Cytogenetic Analysis and Fluorescence In Situ Hybridization}

Chromosome preparations and G-banding were done by standard methods, and fluorescence in situ hybridization (FISH) was done as previously described (Dagenais et al., 1999). For FISH probes, yeast artificial chromosome (YAC) y850A6 was mapped to FRA3B as previously described (Wilke et al., 1994), and YAC y750F1 was obtained from Research Genetics (Huntsville, AL). To obtain a FISH probe specific for FHIT exon 10, a human DNA lambda library was screened with FHIT exon 10 cDNA as a probe by conventional filter hybridization. A $15-\mathrm{kb}$ genomic fragment containing part of intron 9, exon 10, and 3 '-flanking sequence was isolated and used as a probe for FISH.

FISH probes for FHIT intron 5 were obtained by screening of the CITB human bacterial artificial chromosome (BAC) library (Research Genetics) by polymerase chain reaction (PCR) methods using primers within intron 5. Clone 254E1 was identified by screening with PCR primers (5'-GAAGATGAGCAGCGTATGTTGC-3' and 5'-TGTCCTAGGTGCTGAGGACAC-3'), which are approximately 50 $\mathrm{kb}$ distal to exon 5. Clone 385N7 was identified using PGR primers (5'-GGATCTCTCATCGAACCTTCC-3' and 5'-GATCCCTGGGGTGTAACTATC-3'), which are approximately $150 \mathrm{~kb}$ distal to exon 5. Thus, BAC clone 254E1 contains proximal intron 5 sequences, whereas clone $358 \mathrm{~N} 7$ contains sequences from the central region of intron 5 .

Whole-chromosome paint probes to chromosomes 3 (Imagenetics, Framingham, MA), 4 (Oncor, Gaithersburg, MD), and 16 (Oncor) were hybridized separately and in combination according to the manufacturer's instructions. The chromosome 3 probe was directly labeled with Spectrum Orange, and the chromosome 4 and 16 probes were detected with fluorescein isothiocyanate-antidigoxigenin. Chromosomes were counterstained with 4'6-diamidino-2-phenylindole.

\section{Genomic PCR}

Genomic DNA was extracted from the BIC-1 cell line using the QIAamp Tissue Kit (Qiagen, Valencia, CA). PCR was done using markers from FHIT intron 
4 (D3S1481), FHIT intron 5 (D3S4103), and FHIT exon 5 (forward primer: 5'-ATGTCGTTCAGATTTGGCC-3'; reverse primer: 5'-CTGGTACCACAGGTTTCCTA-3').

\section{Reverse Transcription-PCR and Rapid Amplification of cDNA Ends}

Total RNA was isolated from the BIC-1 cell line using Trizol (Gibco BRL, Gaithersburg, MD) according to the manufacturer's instructions. Reverse transcription-PCR (RT-PCR) was done using primers from FHIT exons 1 and 10. Three pairs of primers were used for PCR, with the first pair containing the exon 1 forward primer $\left(5^{\prime}\right.$-CTTTTTGCCGTCTGTTCCCG-3') and exon 1 reverse primer (5'-GGATGTTGACAGCTGGGAATG$\left.3^{\prime}\right)$. The second pair contained the exon 10 forward primer (5'-CGTGAATTCCAGCAAAAGAGC-3') and the exon 10 reverse primer (5'-GCAGCAGAGGAAGGAAGTTTA-3'). The third pair contained the exon 1 forward primer (5'-CTTTTTGCCGTCTGTTCCCG-3') and the exon 10 reverse primer (5'-TCACTGGTTGAAGAATACAGG-3').

We used the 3' RACE system for the rapid amplification of cDNA ends (Gibco BRL). Briefly, first-strand cDNA was reverse-transcribed from 2 $\mu \mathrm{g}$ of total RNA, using the oligo dT-adapter primer supplied by the manufacturer. For 3' RACE, amplification of the target cDNA was done using a forward primer to exon 1 of FHIT (5'-CTTTTTGCCCTCTGTTCCCG-3') and a reverse primer complementary to the adapter. 5' RACE was similarly done using a 5' RACE system (Gibco BRL). First-strand cDNA was reverse-transcribed using a primer specific for FHIT exon 10 (5'-TGCGTGTCTGAGCCGTTTAGGTCTAGG-3'). A poly-C tail then was added to the purified first-strand cDNA in a TdT tailing reaction. Amplification of the target cDNA was performed with the $5^{\prime}$ RACE anchor primer containing poly-G (supplied by the manufacturer) and a nested primer from FHIT exon 10 (5'-TCACTGGRRGAAGAATACAGG$\left.3^{\prime}\right)$. All RACE products were cloned into plasmid vector pCR2.1 (Invitrogen, Carlsbad, CA).

\section{Sequencing}

All sequencing was done by the Sequencing Core Laboratory at the University of Michigan using ABI 377 automated sequencers.

\section{RESULTS}

G-banded chromosome analysis of the early-passage BIC-1 esophageal adenocarcinoma cell line showed a hyperdiploid karyotype with a number of chromosomal rearrangements. The karyotype, as determined by G-banding and FISH with FRA3B probes, is: $48-50, \mathrm{XX},-\mathrm{Y},+\mathrm{X}, \mathrm{t}(3 ; 4)(\mathrm{p} 14.2 ; \mathrm{p} 16)$, $\operatorname{der}(3) \mathrm{t}(3 ; 16)(\mathrm{p} 14.2 ; \mathrm{p} 13.3),-5,+\operatorname{add}(5)(\mathrm{q} 13) \mathrm{x} 2$, $\operatorname{del}(7)(\mathrm{p} 12), \operatorname{der}(8) \mathrm{t}(8 ; 13)(\mathrm{p} 21 ; \mathrm{q} 12), \operatorname{der}(10) \mathrm{t}(10 ;$ 14)(q22;q11)ins(10;?)(q22;?)x2,-14,-14,der(15) $\mathrm{t}(14 ; 15) \quad(\mathrm{q} 11 ; \mathrm{q} 13), \operatorname{der}(16) \mathrm{t}(3 ; 16)(\mathrm{p} 14.2 ; \mathrm{p} 13.3)$ idic (16)(q24)add(16)(p11), add(18)(p11), +3mar[cp6].

Both homologs of chromosome 3 were rearranged, with translocation breakpoints at or near 3 p14.2 and reciprocal breakpoints at 4 p16 and at $16 \mathrm{p} 13$ of an isodicentric chromosome 16. The der(16) had undergone an additional rearrangement, with the presence of additional material of unknown origin translocated to $16 \mathrm{p} 11$ of the other arm of the isodicentric chromosome. RT-PCR amplification of the entire FHIT transcript using primers from exons 1 and 10 resulted in no product, whereas RT-PCR using primers within exons 1 and 10 separately gave fragments of the expected sizes (data not shown). FISH with whole-chromosome paint probes (not shown) indicated a reciprocal translocation between $3 p$ and $\operatorname{der}(16)$ and translocation of chromosome 3 material to $4 \mathrm{p}$. Chromosome 4 or 16 signal was not detected on the other der(3) chromosome with the paint probes used.

These findings led us to look for translocations within the FHIT/FRA3B region using region-specific YAC clones in FISH experiments. YAC y850A6 extends from a region proximal (centromeric) to the FHIT locus to the proximal $\left(5^{\prime}\right)$ end of intron 5. YAC y750F 1 extends from FHIT intron 4 to a region distal to the FHIT locus (Ohta et al., 1996). Both YACs span FRA3B gaps on normal metaphases. Using these YACs as probes on BIC-1 metaphase cells, y750F1 was found to span the translocation breakpoints of both chromosome 3 homologs and showed the reciprocal translocation breakpoints to be at 4p16 and 16p13 (Fig. 1). Because y $750 \mathrm{~F} 1$ extends distally past FHIT, a FHIT exon 10 FISH probe also was used to confirm that the breakpoints were indeed within the FHIT gene (not shown). Exon 10 was translocated to the der(4) and der(16) chromosomes, showing that the breakpoints were between intron 4 and exon 10 of the FHIT locus (not shown).

To define further the location of the breakpoints within the FHIT/FRA3B region on chromosome 3, we performed FISH with BAC clone probes from within the large $(\approx 500 \mathrm{~kb})$ intron 5 of FHIT and genomic PCR with FHIT primers. The results showed that BAC 254E1, containing proximal intron 5 sequences, hybridized only to the $\operatorname{der}(4)$ chromosome, whereas BAC 385N7 from the mid- 
Figure I. Fluorescence in situ hybridization results show translocation breakpoints involving both homologs of chromosome 3. a: YAC y850A6 hybridizes to both $\operatorname{der}(3)$ chromosomes. b: YAC y750FI hybridizes to both $\operatorname{der}(3)$ chromosomes and the $\operatorname{der}(4)$ and $\operatorname{der}(16)$ translocation partners showing that it spans both translocation breakpoints. c: BAC $254 \mathrm{EI}$ hybridizes only to the $\operatorname{der}(4)$ chromosome, showing that it lies distal to one chromosome 3 breakpoint and indicating a proximal intron 5 deletion in the homolog. d: BAC 358 N7 hybridizes to both the $\operatorname{der}(4)$ and $\operatorname{der}(16)$ chromosomes, showing that it lies distal to the breakpoint on both chromosomes 3 .
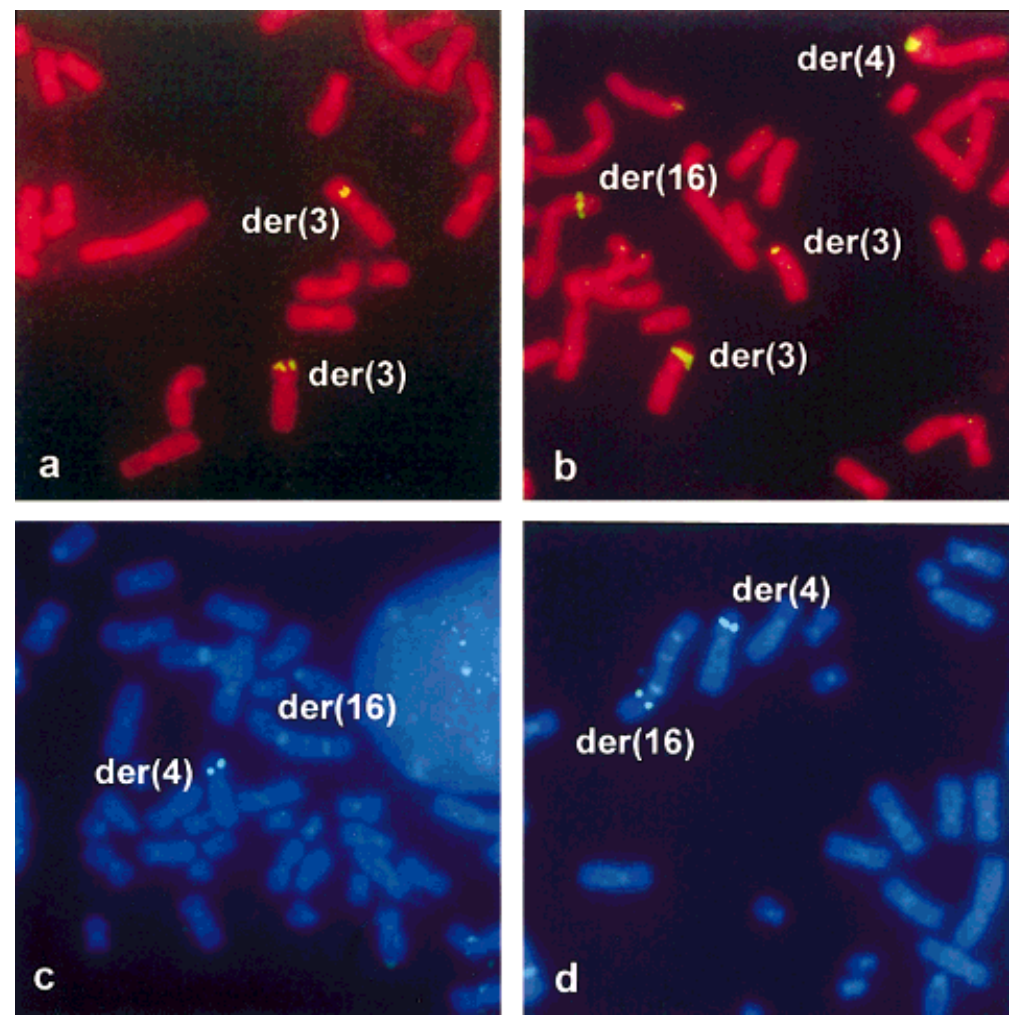

dle of intron 5 hybridized to both the $\operatorname{der}(4)$ and der(16) chromosomes (Fig. 1). Genomic PCR was done using primers from FHIT intron 4 (D3S1481), FHIT intron 5 (D3S4103), and FHIT exon 5. PCR amplification of D3S1481 and D3S4103 gave fragments of the expected sizes. PCR amplification using FHIT exon 5 primers, however, resulted in no fragments, suggesting that exon 5 is homozygously deleted in BIC-1. This deletion was confirmed by Southern blot hybridization of BIC-1 DNA with an exon 5 probe (data not shown).

Taken together, the FISH and genomic PCR data show that both homologs of chromosome 3 are broken in the region from intron 4 to proximal to intron 5, and both translocations are accompanied by different deletions of the region around exon 5 (Fig. 2). The $\mathrm{t}(3 ; 4)$ breakpoint lies proximal to BAC 254E1 and is accompanied by a deletion that encompasses FHIT exon 5 . The $\mathrm{t}(3 ; 16)$ breakpoint is proximal to BAC $385 \mathrm{~N} 7$ and is accompanied by a larger deletion that encompasses exon 5 and the region of intron 5 contained in BAC 254E1. The proximal boundary of both translocations is FHIT intron 4 , or the proximal end of $y 750 \mathrm{~F} 1$, since this YAC spans both translocation breakpoints.

5' RACE from FHIT exon 10 showed only aberrant FHIT transcripts and no chimeric transcripts. The aberrant transcript contained 93 nucleotides of
FHIT intron 8 with FHIT exons 9 and 10. The aberrant transcript may arise from a promoter-like region in intron 8. Using 3' RACE, no transcripts containing chromosome 4 sequences were noted, but novel chimeric transcripts resulting from the $\mathrm{t}(3 ; 16)$ were identified. These contained exons 1, 2, and 4 of the FHIT gene and additional sequences identical to GenBank sequences from BAC clone 185J20, which map to 16p13.3. The chromosome 16 sequences were spliced from three putative exons (TE1, TE2, and TE3), with one chimeric transcript clone containing TE1 and TE3, a second clone containing TE2 and TE3, and a third containing only TE3 (Fig. 3). These three chimeric transcripts appear to result from alternative splicing.

The sequence of the longest 3' RACE product containing FHIT exons 1, 2, and 4 and cryptic exons TE1 and TE3 from 16p13.3 is shown in Figure 4. TE1, TE2, and TE3 contain stop codons in all three reading frames, with the longest open reading frame containing 52 amino acids. Northern blot analysis with TE1, TE2, and TE3 as probes did not detect a positive signal on a human multiple tissue Northern blot (Clontech, Palo Alto, CA) containing brain, heart, skeletal muscle, colon, thymus, spleen, kidney, liver, small intestine, lung, and peripheral blood leukocytes. Thus, TE1-TE3 does not appear to represent a functional gene but 


\section{YAC 750F1}

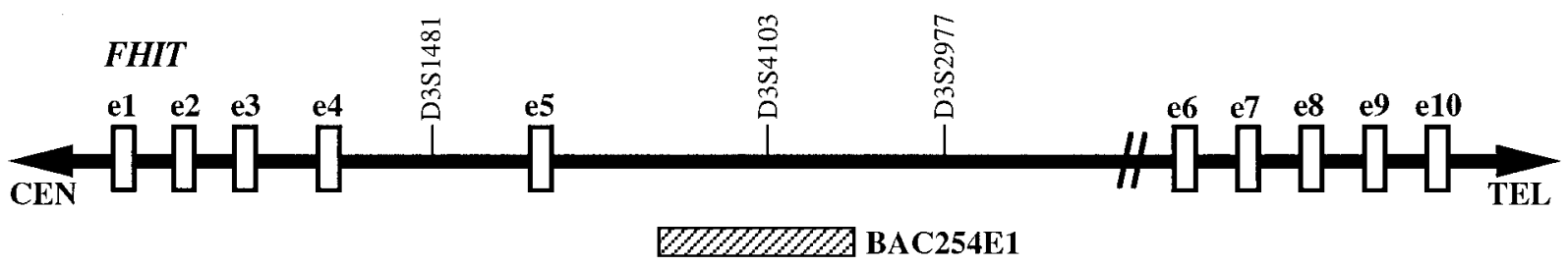

שUWWWWO BAC358N7

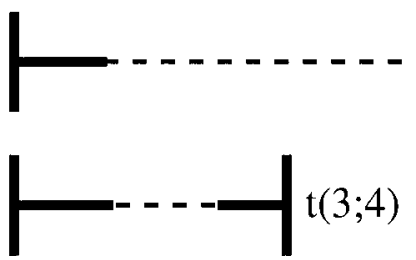

Figure 2. Map of the FHIT locus and position of fluorescence in situ hybridization probes used in this study. The relative positions of the translocation breakpoints are shown, with the deleted regions indicated by the dashed lines.

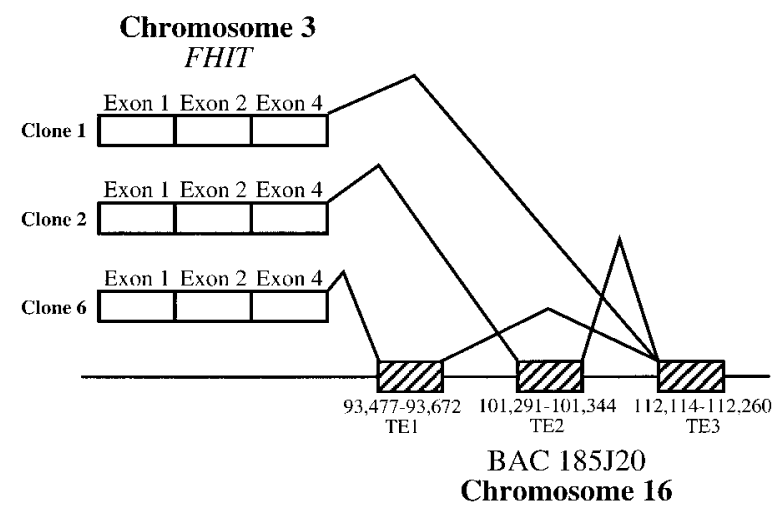

Figure 3. Results of $3^{\prime}$ RACE from FHIT exon I. Three transcripts were identified that contain FHIT exons I, 2, and 4 and additional sequences corresponding to BAC I85J20 from chromosome region $16 \mathrm{p} \mid$ 3.3. The three transcripts appear to result from alternative splicing of the chromosome 16 cryptic exon sequences.

rather a nonfunctional transcribed and spliced region of chromosome 16 resulting from the translocation.

\section{DISCUSSION}

We have shown that both homologs of chromosome 3 are involved in translocations with breakpoints in FHIT/FRA3B and at 4p16 and 16p13.3. All available data indicate that the two translocations are independent events, as opposed to a complex duplication of a single translocation event. The chromosome 3 breakpoints of both translocations are in the region previously shown to be at the center of the FRA3B region, as determined by the position of aphidicolin-induced chromosome gaps on metaphase chromosomes (Smith et al., 1992; Wilke et al., 1994), and are accompanied by deletions encompassing FHIT exon 5. Using RT-PCR, a noncoding chimeric transcript was produced from the $\mathrm{t}(3 ; 16)$ chromosome, which contained FHIT noncoding exons 1, 2, and 4 and three cryptic exon regions from 16p13.3. The RT-PCR results and FISH with whole-chromosome paint probes to chromosomes 3,4 , and 16 indicate that the $\mathrm{t}(3 ; 16)$ was reciprocal. Chromosome 4 material was not identified on the other $\operatorname{der}(3)$ homolog by either FISH or RT-PCR. This finding suggests that the translocation involving $4 \mathrm{p} 16$ was nonreciprocal or, more likely, that the translocation involved only the telomeric region of chromosome 4 and was not detected by the paint probe.

It is now well established that large genomic deletions of up to hundreds of kilobases and aberrant transcripts are a common feature of mutations within the FHIT gene in a variety of tumors (Sozzi et al., 1998). The cells studied here were derived from an esophageal adenocarcinoma, a tumor type with increasing prevalence in the United States (Devesa et al., 1998). We have previously shown that FHIT deletions and aberrant transcripts are an early and common event in esophageal adenocarcinoma and in the precursor metaplastic tissue of 
FHIT exons 1,2 , and 4 TCCCCGCTCTGCTCTGTCCGGTCACAGGACTTTTTGCCCTCTGTTCCCGGGTCCCTCAGGCGGCCACCCAGTGGGCACACTCCCAGGCGGC GCTCCGGCCCCGCGCTCCCTCCCTCTGCCTTTCATTCCCAGCTGTCAACATCCTGGAAGCTTTGAAGCTCAGGAAAGAAGAGAAATCCACT GAGAACAGTCTGTAAAGGTCCGTAGTGCTATCTACATCCAGACGGTGGAAGGGAGAGAAAGAGAAAGAAGGTATCCTAGGAATACCTGCCT TE1 GCTTAGACCCTCTATAAAAGCTCTGTGCATCCTGCCACTGAGGACTCCGAAGAGGTAGCAGTCTTCTGAAAGGGATATGAGGGACCTTTGG

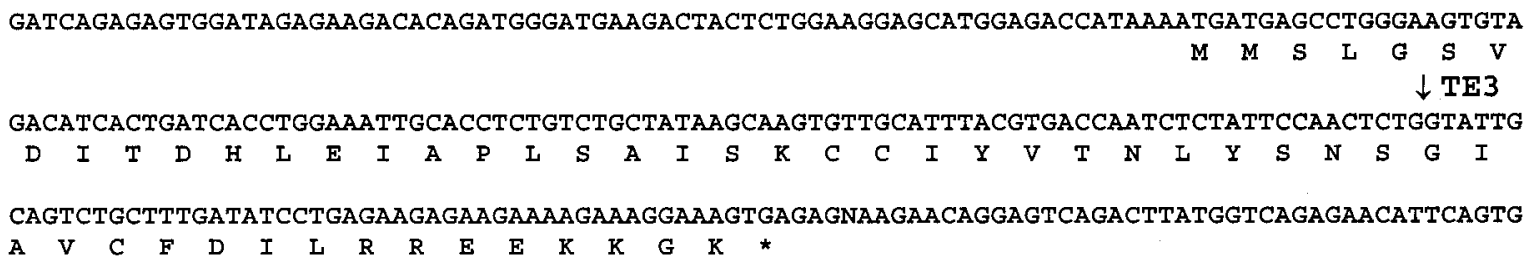

GTGTTGTTGAAATGCCTTTTAAAATAATAAAGAGCTCATGCTCCTAATACTTCAACAAAAAAAAAAAAAAAAAAAAAAAA

Figure 4. Sequence of chimeric transcripts resulting from $t(3 ; 16)$ and identified by $3^{\prime}$ RACE. The longest chimeric transcript containing TEI and TE2 is shown. The $5^{\prime}$ sequence (shaded) contains exons I, 2, and 4 of FHIT. The 3' sequence contains cryptic exon sequences TEI and TE3 (separated by the arrow) from I6p|3.3. The amino acid sequence of the longest open reading frame is shown below the nucleotide sequence.

Barrett esophagus (Michael et al., 1997). The location of FHIT deletions in many tumors largely coincides with the location of FRA3B, as defined by chromosome gaps and breaks on metaphase chromosome preparations. Thus, it is likely that instability of the fragile site is mechanistically responsible for the deletions seen in FHIT in cancer cells. Additional support for this hypothesis comes from recent studies of other cloned fragile site regions. FRA16D (Mangelsdorf et al., 2000; Paige et al., 2000), FRA7G (Huang et al., 1996), and FRAXB (Arlt et al., unpublished observations) have been shown to contain internal deletions in cancer cells or cell lines. Thus, mutation by large deletion appears to be a common feature of common fragile sites in certain tumors.

In contrast to the large number of deletions seen in FRA3B, only one other tumor-associated translocation has been reported to occur in the FHIT/FRA3B region. This is a reciprocal $\mathrm{t}(3 ; 12)(\mathrm{p} 14.2 ; \mathrm{q} 12)$ fortuitously found by examination of a chimeric transcript produced between the HMGIC gene and FHIT 3' exons (Geurts et al., 1997). The findings reported here suggest that these types of translocation events could be more common than is realized at present, since most investigations have looked for deletions in the FHIT/FRA3B region only by PCR or Southern blotting. There are many reported tumor karyotypes with breakpoints in the $3 \mathrm{p} 14$ region. The application of FISH or RT-PCR with FHIT/FRA3B probes and primers to the examination of these and other tumors would allow for a directed study to determine the actual frequency of translocations or similar gross rearrangements.

Common fragile sites have been shown to be frequent sites for translocations in vitro after exposure of cultured cells to aphidicolin and folate stress (Glover and Stein, 1988; Wang et al., 1997). Together with the findings of fragile site-related deletions and instability in tumor cells, this suggests that instability at the fragile site is mechanistically responsible for the translocations reported here and for that shown by Geurts et al. (1997). In both cases, the translocations result in inactivation of the FHIT gene. We also found that both translocations in the BIC-1 cell line were accompanied by relatively large deletions of FHIT exon 5 and proximal intron 5 sequences. The temporal order and connection between the deletion and translocation events cannot be determined, but the FHIT gene could be inactivated by either the deletions or the translocations. As with previously reported FHIT deletions, instability at FRA3B could result in translocations and deletions that may confer a selective advantage for the cells. If the translocation and deletion events occurred sequentially, however, there would be no selection for loss of FHIT function during the latter event.

In summary, we have found both homologs of chromosome 3 to be translocated, with breakpoints in the FRA3B region, in an esophageal adenocarcinoma cell line. This finding lends support to early suggestions regarding common fragile sites and tumor-associated translocations. We suggest that 
translocations involving FRA3B and other common fragile sites, some with the potential for forming chimeric transcripts with other genes, may be more frequent than is realized currently.

\section{ACKNOWLEDGMENTS}

We thank Robert Lyons for sequencing support, Cynthia Gaffney for assistance with manuscript preparation, and Diane Roulston for advice regarding nomenclature. This work was supported by National Cancer Institute grant CA43222 (TWG).

\section{REFERENCES}

Arlt MF, Miller DE, Beer DG, Glover TW. Molecular characterization of FRAXB and comparative common fragile site instability in cancer cells. Human Molecular Genetics, submitted.

Cohen AJ, Li FP, Berg S, Marchetto DJ, Tsai S, Jacobs SC, Brown RS. 1979. Hereditary renal-cell carcinoma associated with a chromosomal translocation. N Engl J Med 301:592-595.

Coquelle A, Pipiras E, Toledo F, Buttin G, Debatisse M. 1997. Expression of fragile sites triggers intrachromosomal mammalian gene amplification and sets boundaries to early amplicons. Cell 89:215-225.

Dagenais SL, Guevara-Fujita M, Loechel R, Burgess AC, Miller DE, Yuzbasiyan-Gurkan V, Brewer GJ, Glover TW. 1999. The canine copper toxicosis locus is not syntenic with ATP7B or ATX1 and maps to a region showing homology to human $2 \mathrm{p} 21$. Mamm Genome 10:753-756.

Devesa SS, Blot WJ, Fraumeni JF. 1998. Changing patterns in the incidence of esophageal and gastric carcinoma. Cancer 83:2049-2053.

Gemmill RM, West JD, Boldog F, Tanaka N, Robinson LJ, Smith DI, Li F, Drabkin HA. 1998. The hereditary renal cell carcinoma 3;8 translocation fuses FHIT to a patched-related gene, TRC8. Proc Natl Acad Sci USA 95:9572-9577.

Geurts JMW, Schoenmakers EFPM, Roijer E, Stenman G, Van de Ven WJM. 1997. Expression of reciprocal hybrid transcripts of $H M G I C$ and FHIT in a pleomorphic adenoma of the parotid gland. Cancer Res 57:13-17.

Glover TW. 1998. Instability at chromosomal fragile sites. Recent Results Cancer Res 154:185-199.

Glover TW, Stein CK. 1987. Induction of sister chromatid exchanges at common fragile sites. Am J Hum Genet 41:882-890.

Glover TW, Stein CK. 1988. Chromosome breakage and recombination at fragile sites. Am J Hum Genet 43:265-273.

Glover TW, Berger C, Coyle J, Echo B. 1984. DNA polymerase $\alpha$ inhibition by aphidicolin induces gaps and breaks at common fragile sites in human chromosomes. Hum Genet 67:136-142.

Hecht F, Glover TW. 1984. Cancer chromosome breakpoints and common fragile sites induced by aphidicolin. Cancer Genet Cytogenet 13:185-188.

Huang H, Reed CP, Mordi A, Lomberk G, Wang L, Shridhar V, Hartmann L, Jenkins R, Smith DI. 1999. Frequent deletions within FRA7G at $7 \mathrm{q} 31.2$ in invasive epithelial ovarian cancer. Genes Chromosom Cancer 24:48-55.

Huang LC, Clarkin KC, Wahl GM. 1996. Sensitivity and selectivity of the DNA damage sensor responsible for activating p53-dependent G1 arrest. Proc Natl Acad Sci USA 93:4827-2832.

Le Beau MM, Rowley JD. 1984. Heritable fragile sites and cancer. Nature 308:607-608.

Mangelsdorf M, Ried K, Woollatt E, Dayan S, Eyre H, Finnis M, Hobson L, Nancarrow J, Venter D, Baker E, Richards RI. 2000. Chromosomal fragile site FRA16D and DNA instability in cancer. Cancer Res 60:1683-1689.

Michael D, Beer DG, Wilke CW, Miller DE, Glover TW. 1997. Frequent deletions of FHIT and FRA3B in Barrett's metaplasia and esophageal adenocarcinomas. Oncogene 15:1653-1659.

Ohta M, Inoue H, Cotticelli MG, Kastury K, Baffa R, Palazzo J, Siprashvili Z, Mori M, McCue P, Druck T, Croce CM, Huebner K. 1996. The FHIT gene, spanning the chromosome 3p14.2 fragile site and renal carcinoma-associated $\mathrm{t}(3 ; 8)$ breakpoint, is abnormal in digestive tract cancers. Cell 84:587-597.

Paige AJW, Taylor KJ, Stewart A, Sgouros JG, Gabra H, Sellar GC, Smyth JF, Porteous DJ, Watson JEV. 2000. A 700-kb physical map of a region of 16q23.2 homozygously deleted in multiple cancers and spanning the common fragile site FRA16D. Cancer Res 60:1690-1697.

Rassool FV, McKeithan TW, Neilly ME, van Melle E, Espinosa R III, Le Beau MM. 1991. Preferential integration of marker DNA into the chromosomal fragile site at 3p14: an approach to cloning fragile sites. Proc Natl Acad Sci USA 88:6657-6661.

Smith PP, Friedman C, Bryant EM, McDougall JK. 1992. Viral integration and fragile sites in human papillomavirus-immortalized human keratinocyte cell lines. Genes Chromosomes Cancer 5:150-157.

Sozzi G, Huebner K, Croce CM. 1998. FHIT in human cancer. Adv Cancer Res 74:141-166.

Wang L, Paradee W, Mullins C, Shridhar R, Rosati R, Wilke CM, Glover TW, Smith DI. 1997. Aphidicolin-induced FRA3B breakpoints cluster in two distinct regions. Genomics 41:485-488.

Wilke CM, Guo SW, Hall BK, Boldog F, Gemmill RM, Chandrasekharappa SC, Barcroft CL, Drabkin HA, Glover TW. 1994. Multicolor FISH mapping of YAC clones in 3p14 and identification of a YAC spanning both FRA3B and the $t(3 ; 8)$ associated with hereditary renal cell carcinoma. Genomics 22:319-326.

Yunis JJ, Soreng AL. 1984. Constitutive fragile sites and cancer. Science 226:1199-1204 